Research Article

Yousif Atyeib Ibrahim Hassan*

\title{
Structures of spinors fiber bundles with special relativity of Dirac operator using the Clifford algebra
}

https://doi.org/10.1515/dema-2021-0035

received March 11, 2021; accepted June 6, 2021

Abstract: The purpose of this article is to demonstrate how to use the mathematics of spinor bundles and their category. We have used the methods of principle fiber bundles obey thorough solid harmonic treatment of pseudo-Riemannian manifolds and spinor structures with Clifford algebras, which couple with Dirac operator to study important applications in cohomology theory.

Keywords: Clifford and spinor bundles, principle bundle, spin structures, Dirac operator

MSC 2020: 55N08, 55R05, 55R20

\section{Introduction}

The interplay between physics and mathematics has spurred each of the disciplines to great heights. It has led to numerous discoveries, not the least of which is the Dirac operator and the related concept of the spinor. The first groundwork for these concepts was laid down by Clifford in the middle of the nineteenth century as a generalization of the quaternions of Hamilton and the exterior algebra [1] of Grassmann. In 1913 [1], Elie Cartan wrote down the general theory of spinors. Spinors were first applied to mathematical physics by Wolfgang Pauli in 1927 [1], when he introduced his spin matrices. On the physics, Dirac introduced his famed operator in 1928 [1,2] but made no mention of the connection with spinors, and this was only done much later. During the years 1940-1970, the spinors and Clifford algebra of bundle became a fundamental tool of particle physics and came back later, at the forefront of differential geometry and of mathematics in general, with the recognition of the importance of the Dirac operator and theory of spinors in differential geometry.

Connections on fiber bundles and their relation with covariant derivation is discussed. In the final section, spin structures are introduced as nontrivial coverings of SO bundles, followed by a proof of necessary and the sufficient condition for their existence in terms of SO bundle and Dirac operator.

\section{Clifford algebras}

A Clifford algebra is a type of associative algebra, which can be considered a generalization of the usual associative fields such as $\mathbb{R}, \mathbb{C}$ or $\mathbb{H}$. In fact, these fields can be seen as particular examples of Clifford

\footnotetext{
* Corresponding author: Yousif Atyeib Ibrahim Hassan, Department of Mathematics, College of Science and Arts, Qassim University, ArRass, Kingdom of Saudi Arabia, e-mail: y.hassan@qu.edu.sa
} 
algebras [1]. It is defined over a vector space $V$ over a field $k$ denoted by $V_{k}$ equipped with a quadratic form $q$ defined as follows:

Definition 1. A quadratic form $q$ is an operator from a vector space $V_{k}$ to its fields $k$, such that if $\alpha \in k$ and $\delta$, $\eta \in V_{k}$, then $q(\alpha \eta)=\alpha^{2} q(\eta)$ and $2 q(\delta, \eta)=q(\delta+\eta)-q(\delta)-q(\eta)$ is a symmetric bilinear form on $V_{k}$ [2]. A nondegenerate quadratic form is a quadratic form with the extra condition that $q(\eta)=0 \Leftrightarrow \eta=0$ [1].

A Clifford algebra is a generalization of the exterior or Grassmann algebra precisely.

Definition 2. Let $V_{k}$ be a vector field over a field $k$ and take the tensor algebra $T\left(V_{k}\right)=\sum_{i=0}^{\infty} \oplus^{i} V_{k}=k \oplus V_{k} \oplus$ $V_{k} \otimes V_{k} \oplus \ldots$ and let $q$ be a quadratic form [1]. A Clifford algebra $\mathrm{Cl}\left(V_{k}, q\right)$ on $V_{k}$ is then $\operatorname{Ce}\left(V_{k}, q\right)=T\left(V_{k}\right) / I\left(V_{k}\right.$, $q$ ) with $I\left(V_{k}, q\right)$, the two-sided ideal generated by $\eta \otimes \eta+q(\eta)[1,2]$. This is an associative algebra with unit, the exterior algebra can be related with the Clifford algebra with quadratic form 0. Defining the Clifford algebra and deriving its basic properties, we follow [2], but we could have equivalently started with the universal property proven below and worked our way back from there.

Lemma 1. Every vector space with a quadratic form has a $q$-orthogonal basis $\left\{e_{1}, \ldots, e_{\mathrm{n}}\right\}$, that is, $q\left(e_{i}, e_{j}\right)=0$ if $i \neq j[1]$.

Definition 3. (Pin and Spin groups)

The group Pin is the following subgroup of the Clifford group:

$$
\operatorname{Pin}\left(V_{k}, q\right)=\left\{\eta \in \check{P}\left(V_{k}, q\right) \mid q(\eta)= \pm 1\right\}
$$

i.e., we define $P\left(V_{k}, q\right) \subset \check{P}\left(V_{k}, q\right)$ (where $\check{P}\left(V_{k}, q\right)$ calls Lipschitz group which is "largest" spinor group) to be the subgroup of $\mathrm{Cl}\left(V_{k}, q\right)$ generated by the elements $\eta \in V_{k}$ with $q(\eta) \neq 0$. And that there is a representation

$$
P\left(V_{k}, q\right) \stackrel{\mathrm{Ad}}{\longrightarrow} O\left(V_{k}, q\right)
$$

The group spin is the even subgroup of $\operatorname{Pin}: \operatorname{Spin}\left(V_{k}, q\right)=\operatorname{Pin}\left(V_{k}, q\right) \cap \mathrm{Cl}^{\mathrm{O}}\left(V_{k}, q\right)[1,2]$.

Theorem 2. Let $k$ be a spin field. Then, $\operatorname{Pin}\left(V_{k}, q\right)$ is the kernel of $N: \check{P}\left(V_{k}, q\right) \rightarrow k^{\times}$, (where $k^{\times}$is nonzero multiples of 1) and the twisted adjoint $\left.\widetilde{\mathrm{Ad}}\right|_{\operatorname{Pin}\left(V_{k}, q\right)}$ is a surjection of $\operatorname{Pin}\left(V_{k}, q\right)$ on $O\left(V_{k}, q\right)$. Then, the sequence:

$$
1 \rightarrow \mu_{4}(k) \rightarrow \operatorname{Pin}\left(V_{k}, q\right) \stackrel{\widehat{\mathrm{Ad}}}{\longrightarrow} O\left(V_{k}, q\right) \rightarrow 1
$$

with $\mu_{4}(k)$ the fourth roots of 1 in the field. For example, $\mu_{4}(\mathbb{R})=\{-1,1\} \approx \mathbb{Z}_{2}$. And $\mu_{4}(\mathbb{C})=\{-1,1,-i, i\} \approx \mathbb{Z}_{4}^{(1,10)}$.

Proof. See [1].

We can also consider the covering group of $\operatorname{SO}\left(V_{k}, q\right)=\left\{x \in O\left(V_{k}, q\right) \mid \operatorname{det}(x)=1\right\}$. Then, we have the following:

Corollary 1. The group $\operatorname{Spin}\left(V_{k}, q\right)$ is a cover of $\mathrm{SO}\left(V_{k}, q\right)$, and we have the exact sequence:

$$
1 \rightarrow \mu_{4}(k) \rightarrow \operatorname{Spin}\left(V_{k}, q\right) \stackrel{\widetilde{\mathrm{Ad}}}{\longrightarrow} \mathrm{SO}\left(V_{k}, q\right) \rightarrow 1
$$

with $\mu_{4}(k)$ as in Theorem $2[1,3]$.

Proof. Let $x \in \operatorname{Pin}\left(V_{k}, q\right)$. Then, $\widetilde{\operatorname{Ad}}_{x}$ is equal to the composition of a number of reflections due to Theorem 2 . We have that for all $v \in V_{k}$, $\operatorname{det}\left(\widetilde{\mathrm{Ad}}_{\eta}\right)=-1$. To see this, take a $q$-orthogonal basis with $\eta_{1}=\eta$ and $q\left(\eta, \eta_{j}\right)=0$ for all $j>1$. Then, $\widetilde{\operatorname{Ad}_{\eta}}\left(\eta_{1}\right)=-\eta$ by definition and $\widetilde{\operatorname{Ad}}_{\eta}\left(\eta_{j}\right)=\eta_{j}$ with $j>1$ by $\operatorname{definition}$, so $\operatorname{det}\left(\widetilde{\operatorname{Ad}}_{\eta}\right)=-1$. 
So any element of $\operatorname{SO}\left(V_{k}, q\right)$ is generated by an even number of reflection, and so $\operatorname{Spin}\left(V_{k}, q\right)$ must be generated by an even number of vectors, so by the properties of the $\mathbb{Z}_{2}$ grading we get that it is also an element of $\mathrm{Cl}^{O}\left(V_{k}, q\right)$. The exact sequence follows immediately from Theorem 2 [1].

Proposition 3. For any $x$ in $\operatorname{Spin}(n)$, there exists an even integer $p$ and elements $f_{1}, \ldots, f_{p}$ of norm 1 such that $x=f_{1}, \ldots, f_{p}$. The reverse statement also holds [4].

Theorem 4. For any $n \geq 2$, $\operatorname{Spin}(n)$ is connected. For $n \geq 3$, $\operatorname{Spin}(n)$ is simply connected; hence, $\operatorname{Spin}(n)$ is the universal covering Lie group of $\mathrm{SO}(n)$.

Proof. Then, the short exact sequence of Lie groups.

$$
1 \rightarrow \mu_{4}(k) \rightarrow \operatorname{Spin}\left(V_{k}, q\right) \stackrel{\widetilde{\mathrm{Ad}}}{\longrightarrow} \mathrm{SO}\left(V_{k}, q\right) \rightarrow 1 .
$$

Since $\pi_{1} \mathrm{SO}(n)=\mathbb{Z}_{2}$ for any $n \geq 3$, any connected couple covering of $\mathrm{SO}(n)$ is simply connected for $n \geq 3$. Hence, it suffices to show that $\operatorname{Spin}(n)$ is connected for any $n \geq 2$ [3].

First, let us show that 1 and -1 are path connected in $\operatorname{Spin}(n)$. Since the dimension is at least 2 , there are elements $e_{1}$ and $e_{2}$ in $V_{k}$, such that $\left\|e_{1}\right\|=\left\|e_{2}\right\|=1$ and $\left\langle e_{1}, e_{2}\right\rangle=0$. For $0 \leq t \leq \pi$, we define $\gamma(t)=\cos t+$ $e_{1} e_{2} \sin t=e_{1}\left(-e_{1} \cos t+e_{2} \sin t\right)$. It is trivial that the norm $\left(-e_{1} \cos t+e_{2} \sin t\right)$ is 1 . By Proposition $3, \gamma(t)$ is an element of $\operatorname{Spin}(n)$ for any $t$. We have $\gamma(0)=1$ and $\gamma(\pi)=-1$.

Second, note that any element $y$ of $\operatorname{Spin}(n)$ can be connected with $y$ by the path $y y(t)$.

Finally, let $x$ and $y$ be in $\operatorname{Spin}(n)$. Since $\mathrm{SO}(n)$ is connected, there exists a path from $\rho(x)$ to $\rho(y)$ in $\mathrm{SO}(n)$. We may lift this path to a path starting in $x$ and ending in a point $y^{\prime}$ of $\operatorname{Spin}(n)$. Since the kernel of $\rho$ equals $\{ \pm 1\}$, it holds that $y^{\prime}=y$ or $y^{\prime}=-y$. In the first case, we are done. In the second case, we connect $x$ with $-y$ and then connect $-y$ with $y$ [4].

We now can construct any real Clifford algebra we are interested in. For example, the physically interesting Clifford algebras $\mathrm{Cl}_{1,3}$ and $\mathrm{Cl}_{3,1}$ are now constructed as $\mathrm{Cl}_{1,3} \simeq \mathrm{Cl}_{0,2} \otimes \mathrm{Cl}_{1,1}=\mathbb{H} \otimes \mathbb{R}(2) \simeq \mathbb{H}(2)$ and $\mathrm{Cl}_{3,1} \simeq \mathrm{Cl}_{2,0} \otimes \mathrm{Cl}_{1,1}=\mathbb{R}(2) \otimes \mathbb{R}(2) \simeq \mathbb{R}(4)[1]$.

Theorem 5. There are isomorphisms

$$
\begin{aligned}
& \mathrm{Cl}_{n, 0} \otimes \mathrm{Cl}_{0,2} \simeq \mathrm{Cl}_{0, n+2}, \\
& \mathrm{Cl}_{0, n} \otimes \mathrm{Cl}_{2,0} \simeq \mathrm{Cl}_{n+2,0}, \\
& \mathrm{Cl}_{r, s} \otimes \mathrm{Cl}_{1,1} \simeq \mathrm{Cl}_{r+1, s+1},
\end{aligned}
$$

or all $n, r, s \geq 0$ [2].

Note that here we are using the ungraded tensor product.

Proof. See [1].

Remark 1. It is standard notation to write: $q_{r, s} \equiv q, O_{r . s} \equiv O\left(V_{k}, q\right)$ and $\mathrm{SO}_{r, s} \equiv \mathrm{SO}\left(V_{k}, q\right)$. In accordance, we write $\operatorname{Pin}_{r, s} \equiv \operatorname{Pin}\left(V_{k}, q\right)$ and $\operatorname{Spin}_{r, s} \equiv \operatorname{Spin}\left(V_{k}, q\right)$. Similarly, it is conventional to write $O_{n} \equiv O_{n, O} \cong O_{O, n}$ and $\mathrm{SO}_{n} \equiv \mathrm{SO}_{n, O} \cong \mathrm{SO}_{O, n}$. Thus, we set $\operatorname{Pin}_{n}=\operatorname{Pin}_{n, o}$, and $\operatorname{Spin}_{n}=\operatorname{Spin}_{n, O}$.

We also write $P_{r . s} \equiv P\left(V_{k}, q\right)$ and $\check{P}_{r, s} \equiv \check{P}\left(V_{k}, q\right)[1]$.

Definition 4. A section of Clifford $\mathrm{C} \ell\left(V_{k}, q\right)$ is called Clifford field [5]. 


\section{Spinors}

We will build representations with $m$-dimensions of spinor on a complex vector, and it will become clear that the complex Clifford algebra has a much simpler structure than the real one, a period of degree 2 instead of degree 8 as in the real case [3]. Now if $m$ is even, $m=2 n$, we have Welly representation, which restricts to $\mathrm{Cl}^{\mathrm{O}}\left(V_{k}, q\right)$.

Definition 5. Clifford's complex algebra $\mathrm{C}^{\mathbb{C}}\left(V_{k}, Q\right)$ is a Clifford algebra that is constructed by starting with a complex vector space $V_{k} \otimes_{\mathbb{R}} \mathbb{C}$, and $Q$ extends through the complex linearity and then using the definition as real case. By starting with a real vector space $V_{k}$ of dimension $n$, then this is denoted by $\mathrm{Cl}^{\mathbb{C}}(n)$. One can easily see that $\mathrm{Cl}^{\mathbb{C}}(n)=\mathrm{Cl}(n) \otimes C \ell$. Build a spin representation as being reversible elements in $\mathrm{Cl}(n)$ are complexified, producing a structure of $\operatorname{Spin}(n, \mathbb{C})$. (The complexification of $\operatorname{Spin}(n)$ ) is a reversible element in $\mathrm{Cl}^{\mathbb{C}}(n)$.)

By an inductive argument. The algebras $\mathrm{Cl}^{\mathbb{C}}(n)$ constructed to begin

$$
\mathrm{Cl}(1)=\mathbb{C}, \mathrm{Cl}(2)=\mathbb{H},
$$

so

$$
\mathrm{Cl}^{\mathbb{C}}(1)=\mathrm{C} \ell(1) \otimes_{\mathbb{R}} \mathbb{C}=\mathbb{C} \oplus \mathbb{C}
$$

and

$$
\mathrm{Cl}^{\mathbb{C}}(2)=\mathrm{C} \ell(2) \otimes_{\mathbb{R}} \mathbb{C}=\mathbb{H} \oplus_{\mathbb{R}} \mathbb{C}=M(2, \mathbb{C}) .
$$

Theorem 6. $\mathrm{Cl}^{\mathbb{C}}(n+2)=\mathrm{C} \ell^{\mathbb{C}}(n) \otimes_{\mathbb{C}} C \ell^{\mathbb{C}}(2)=\mathrm{C} \ell^{\mathbb{C}}(n) \otimes_{\mathbb{C}} M(2, \mathbb{C})$.

To start the induction, we put $n=1,2$.

Corollary 2. If $n=2 k, C e^{\mathbb{C}}(2 k)=M(2, \mathbb{C}) \otimes \cdots \otimes M(2, \mathbb{C})=M\left(2^{K}, \mathbb{C}\right)$, where the product has $k$ factors, and if $n=2 k+1, C \ell^{\mathbb{C}}(2 k+1)=C \ell^{\mathbb{C}}(1) \otimes M\left(2^{k}, \mathbb{C}\right)=M\left(2^{K}, \mathbb{C}\right) \oplus M\left(2^{K}, \mathbb{C}\right)[3]$.

Proof. Choose generators $g_{1}, g_{2}$ of $\mathrm{Cl}(2), f_{1}, \ldots, f_{n}$ of $\mathrm{Cl}(n)$ and $\breve{e}_{1}, \ldots, \breve{e}_{n+2}$ of $\mathrm{Cl}(n+2)$. Then, we get the symmetry by the following map:

$$
\begin{gathered}
\breve{e}_{1} \rightarrow 1 \otimes g_{1} \\
\breve{e}_{2} \rightarrow 1 \otimes g_{2} \\
\breve{e}_{3} \rightarrow \mathrm{if}_{1} \otimes g_{1} g_{2} \\
\vdots \\
e_{n+2} \rightarrow \mathrm{if}_{n} \otimes g_{1} g_{2}
\end{gathered}
$$

If $n=2 k$ (even case). In this case, Clifford's complex algebra is the algebra of $2^{k}$ by $2^{k}$ complex matrices.

Definition 6. A spin structure on a pseudo-Riemannian vector bundle with signature $(r, s) E$ is a principal $\operatorname{Spin}(r, s)$ bundle $P_{\text {Spin }}(E)$ together with a two-sheeted covering $\xi: P_{\mathrm{Spin}}(E) \rightarrow P_{\mathrm{SO}}(E)$, such that $\xi(p g)=$ $\xi(p) \xi_{0}(g)$ for all $p \in P_{\text {Spin }}$ and $g \in \operatorname{Spin}(r, s)$ and $\xi_{0}$ the covering map $\operatorname{Spin}(r, s) \rightarrow \operatorname{SO}(r, s)[2,3]$.

Two spin structures $P_{\mathrm{Spin}}(E)$ and $P_{\mathrm{Spin}}^{\prime}(E)$ are called equivalent if there is a mapping $F$, such that $F(g h)=$ $F(g) h$ for $g \in P_{\text {Spin }}$ and $h \in$ Spin and the following diagram commutes:

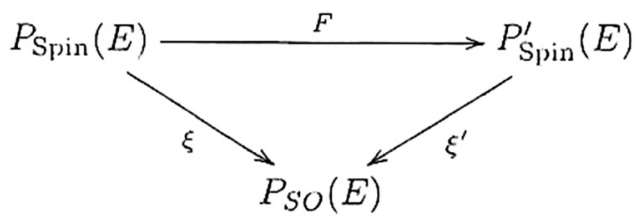


This means that if they are equivalent as spin structures, they are equivalent as principal fiber bundles [1,2].

From now on, we will only consider $r, s$ such that $\pi_{1}(\mathrm{SO}(r, s))=\mathbb{Z}_{2}$. This makes many proofs much simpler since it makes $\operatorname{Spin}_{(r, s)}$ simply connected. Also, this situation is the one where most examples interesting to physics are, when $r \geq 3$ and $s=0$ or 1, or vice-versa. The definition of the spin structure gives the following commutative diagram:

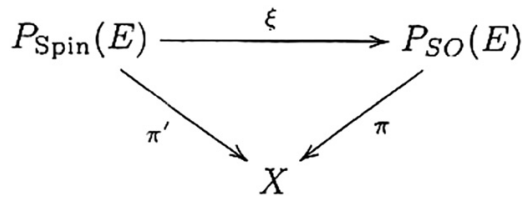

Since restriction to fibers gives the covering map $\xi_{0}$, this diagram can be extended [1,2]:

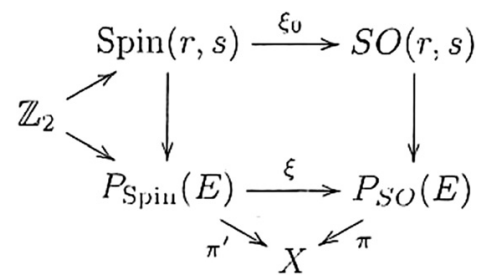

In the above figure, we see that the vertical lines show the inclusions of fibers in the fiber bundle. Now we can consider whether given a two-sheeted covering $\varphi: C_{2}(E) \rightarrow P_{\mathrm{SO}}(E)$ of $P_{\mathrm{SO}}(E)$ gives a spin structure. It certainly gives a fiber bundle over $E$ since we can set $\pi^{\prime}=\pi_{\circ} \xi$.

Now we see that this bundle gives a spin structure if the covering is nontrivial on the fibers.

Theorem 7. If $\pi_{1}(\mathrm{SO}(r, s))=\mathbb{Z}_{2}$, then the spin structures are in one-to-one correspondence with two-sheeted coverings of $P_{\mathrm{SO}}(E)$, which are nontrivial on the fibers $[1,2]$.

Now consider a spin structure $\xi: P_{\text {Spin }} \rightarrow P_{\text {SO }}$. Define $\alpha_{F} \in \pi_{1}(\mathrm{SO}(r, s))$, the nontrivial element. The spin structure $\xi$ induces a group homomorphism $\xi_{*}: \pi_{1}(P) \rightarrow \pi_{1}(Q)$. This subgroup of $\pi_{1}(Q)$ is a subgroup of index 2 because of covering morphism, and $P_{\text {Spin }}$ is a double covering of $P_{\text {So }}$ fiber wise.

\section{Lemma 8.}

$$
\alpha_{F} \notin \xi_{*}\left(\pi_{1}\left(P_{\text {Spin }}\right)\right)
$$

Proof. Suppose $\alpha_{F} \in \xi_{*}\left(\pi_{1}\left(P_{\text {Spin }}\right)\right)$. Then, the inclusion map $i$ : SO $\rightarrow P_{\text {SO }}$ lifts to a continuous map.

$I: \mathrm{SO} \rightarrow P_{\text {Spin }}$ such that

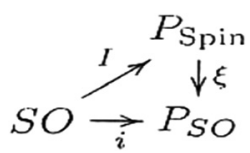

commutes. $I$ (SO) $\subset P_{\text {Spin }}$ is contained in one fiber Spin of $P_{\text {Spin }}$, so $I: \mathrm{SO}(r, s) \rightarrow \operatorname{Spin}_{(r, s)}$ with $\xi_{0} \circ I=\operatorname{IdSO}(r, s)=$ $\operatorname{Id} \mathbb{Z}_{2}$. Then, $\xi_{0 *} I_{*}=\operatorname{Id} \pi_{1}(\mathrm{SO}(r, s))$ and $\pi_{1}(\mathrm{SO}(r, s))=\mathbb{Z}_{2}$ and $\pi_{1}(\operatorname{Spin}(r, s))=1$, so we get a contradiction, so $\alpha_{F} \notin \xi_{*}\left(\pi_{1}\left(P_{\text {Spin }}\right)\right)[6]$.

This lemma is then used to prove a classification theorem of spin structures, following and using the classification of covering spaces. 
Theorem 9. $A \mathrm{SO}(r, s)$-principal fiber bundle $P_{\mathrm{SO}}$ over a manifold $M$ has a spin structure if and only if there is a short split exact sequence.

$$
0 \rightarrow \mathbf{Z}_{2} \stackrel{i_{*}}{\longrightarrow} \pi_{1}(\mathrm{PSO}) \stackrel{\pi_{*}}{\longrightarrow} \pi_{1}(M) \rightarrow 0
$$

meaning that $\pi_{1}\left(P_{\mathrm{SO}}\right)$ is isomorphic to $K \times \mathbb{Z}_{2}$ and $\pi_{*}$, the map induced by projection map, maps $K$ isomorphically to $\pi_{1}(M)$.

To prove this theorem, we need a few lemmas. First, we prove the necessity of the conditions, we assume $P_{\text {SO }}$ has a spin structure $P_{\text {Spin }}$.

Lemma 10. The map $\pi_{*}^{\prime}: \pi_{1}\left(P_{\text {Spin }}\right) \rightarrow \pi_{1}(M)$ is an isomorphism.

Proof. First, we prove surjectivity. Take an element $[g] \in \pi_{1}(M)$ represented by a loop $g:[0,1] \rightarrow M$ based at $m_{0} \in M$. We can then look at a path $\tilde{g}:[0,1] \rightarrow P_{\text {Spin }}$ such that $\pi \tilde{g}(t)=g(t)$. This path exists because $P_{\text {Spin }}$ is a fiber bundle over $M$. This path does not have to be a loop. However, $\pi(\tilde{g}(0))=\pi(\tilde{g}(1))=m_{0}$ and the fibers of $P_{\text {Spin }}$ are path connected, so we can find a path $\delta$ in spin such that $\delta(0)=1$ and $\delta(1) \tilde{g}(1)=g(0)$. The product path $\delta \tilde{g}$ is now a loop and $\pi(\delta \tilde{g})=g$. This proves surjectivity.

As for injectivity, consider a loop $\tilde{h}:[0,1] \rightarrow P_{\text {Spin }}$ and assume $\pi(\tilde{h})(t)=h(t)$ is homotopic to trivial loop, say at the point $x_{O}=h(0)$. There is then a homotopy from the loop $h$ to the point $x_{0}$. This homotopy can be covered by a homotopy of $\tilde{h}$ into a new loop lying in the fiber $\pi^{-1}\left(x_{0}\right)$, or its generalization to fiber bundles over paracompact spaces [7]. Since spin is simply connected, there is then a homotopy to a single point in this fiber; hence, $\tilde{h}$ is homotopic to the trivial loop, so $\pi_{*}$ is injective.

Lemma 11. The map $\pi_{*}: \pi_{1}\left(P_{\mathrm{SO}}\right) \rightarrow \pi_{1}(M)$ restricted to the image of $\xi_{*}\left(\pi_{1}\left(P_{\mathrm{Spin}}\right)\right)$ is an isomorphism.

Proof. Call the image of $\xi_{*} K$. We have the following commutative diagram:

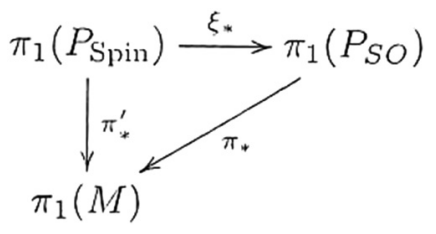

The map $\xi_{*}$ is injective, and according to the covering space, morphism is injective. We also know that $\pi^{\prime}$ is an isomorphism, so $\pi_{*} \mid k$ is injective. Furthermore, $\pi_{*} \mid k \circ \xi_{*}=\pi^{\prime}$ is an isomorphism, so $\pi_{*} \mid k$ must be surjective, hence an isomorphism [1].

Lemma 12. The map $i_{*}: \pi_{1}(\mathrm{SO})=\mathbb{Z}_{2} \rightarrow \pi_{1}\left(P_{\mathrm{SO}}\right)$ is injective, so it is an isomorphism onto its image.

Proof. Suppose $[g] \in \pi_{1}(\mathrm{SO})$ and $i_{*}[g]=[\alpha] \in \pi_{1}\left(P_{\mathrm{SO}}\right)$. If $[\alpha]$ is trivial, then we can lift $\alpha$ to a path $\hat{\alpha}$ in $P_{\text {Spin }}$. The loop $\alpha$ lies within a single fiber, so the loop $\hat{\alpha}$ also does. Because Spin is simply connected, there is a homotopy between $\hat{\alpha}$ and the trivial loop within the fiber. Applying the covering map to of $P_{\mathrm{SO}}$, SO, so [ $g$ ] is trivial and $i_{*}$ is injective.

Lemma 13. The sequence of Lemma 11 is exact, in particular: $\operatorname{ker}\left(\pi_{*}\right)=\operatorname{img}\left(i_{*}\right)[1,2]$.

Proof. Take a $[g] \in \pi_{1}(\mathrm{SO})$. Then, $i_{*}[g]$ has a representative lying within a single fiber. Then, $\pi_{*} i_{*}[g]=[e]$ and hence, $\operatorname{im}\left(i_{*}\right) \subseteq \operatorname{ker}\left(\pi_{*}\right)$. Take a loop $\alpha \subseteq P_{\text {SO }}$. We will construct a loop $\hat{\alpha} \subset i\left(P_{\text {Spin }}\right)$ such that $\alpha=\hat{\alpha} g$ with $g$ a loop in SO. First, let $\alpha^{\prime}=\pi_{\circ} \alpha$ be a loop in $M$. Then, we lift this loop one in $P_{\text {Spin }}$ by lifting and multiplying with a path $\delta$ with $\delta(0)=1$ and $\alpha^{\prime}(1) \delta(1)=\alpha(0)$. Now define $\hat{\alpha}=\xi\left(\alpha^{\prime} \delta\right)$. We can now conclude that $[\hat{\alpha}]=\xi_{*} \pi_{*}^{\prime-1} \pi_{*}([\alpha])$ and that $\pi(\alpha)=\pi(\hat{\alpha})$. Thus, we have that $\alpha=\hat{\alpha} g$ for some loop $g \subseteq$ SO; hence, $[\alpha]=$ 
$[\hat{\alpha} g]=[\hat{\alpha}] i_{*}[g]$, so if $[\alpha] \in \operatorname{ker}\left(\pi_{*}\right)$ we have that because $\pi_{*}$ is an isomorphism on the image of $\xi_{*}$, that $[\alpha]=$ $[\hat{\alpha}] i_{*}[g]=[e] i_{*}[g], \operatorname{so} \operatorname{ker}\left(\pi_{*}\right) \subseteq \operatorname{im}\left(i_{*}\right)[1]$.

If the fundamental group of $\mathrm{SO}(r, s)$ is not $\mathbb{Z}_{2}$, this result can be generalized, but the double covering space of $\mathrm{SO}(r, s)$ will not be a universal covering space.

Instead, one must look at either the universal covering space of $\mathrm{SO}(r, s)$, which is then not equal to $\operatorname{Spin}_{(r, s)}$, or one must look at not simply connected $\operatorname{Spin}_{(r, s)}[1,6]$.

The condition of Lemma 11 for the existence of a spin structure can be shown to be equivalent with the usual condition that the second Stiefel-Whitney class $w_{2}$ of $M$ vanishes [1,6,8]. For the special case of $\mathrm{SO}_{0}(1,3)$-principal fiber bundles over a noncompact 4-manifold $M$, the case in general relativity, it has been shown that any existing spin structures are trivial, $M \times \operatorname{Spin}(1,3)$ and so it has a spin structure, if and only if the $\mathrm{SO}_{0}(1,3)$-bundle is parallelizable, meaning that there is a global section of the $\mathrm{SO}_{0}(1,3)$ bundle [1].

Definition 7. We call global sections of $P_{\mathrm{SO}(1,3)}(M)$ Lorentz frames and global sections of $P_{\mathrm{Spin}(1,3)}(M)$ spin frames [5].

Remark 2. When we will use the concept of the spin structure in physics, the space-time is fourdimensional manifold with a metric of signature $(3,1)$ or $(1,3)$, we will naturally use $\operatorname{SL}(2, \mathbb{C})$ as group instead of $\operatorname{Spin}(p, q)$.

The isomorphism $S L(2, \mathbb{C}) \simeq \operatorname{Spin}(1,3)$ [9]. Here, the fundamental interactions of spinors, Dirac, Weyl spinors (half-spinors), Majorana spinors, and bi-quaternions are used to describe the most fundamental particles in same dimension.

\section{Clifford algebras and spinor bundles}

There are two equivalent ways of defining a Clifford bundle of a pseudo-Riemannian vector bundle $E$ over a manifold $X$. One is the obvious generalization of $C \ell\left(\mathbb{R}^{n}\right)$ :

$$
\mathrm{Ce}(E)=\bigcup_{\mathrm{x} \in \mathrm{X}} \mathrm{Ce}\left(E_{x}, q_{x}\right) .
$$

where $q$ is a smooth quadratic form on $E$ and $q_{x}$ is the restriction of that form to the fiber over $x$. This definition emphasizes that the Clifford bundle is a bundle of Clifford algebra's over $X$. The other definition uses associated bundles and can be used to determine the topology, as follows: An orthogonal transformation with respect to an inner product of signature $(r, s)$ in $\mathbb{R}^{(r+s)}$ induces an orthogonal transformation in $\mathrm{Cl}_{r, s}$, since it preserves the ideal. This induced map preserves the multiplication in $\mathrm{Cl}_{r, s}$, so if we take an orthogonal transformation $\rho_{r, s}$ from $\mathrm{SO}(r, s)$, we get a map $\operatorname{cl}\left(\rho_{r, s}\right): \mathrm{SO}_{r, s} \rightarrow \operatorname{Aut}\left(\mathrm{Cl}_{r, s}\right)$. The bundle associated to this bundle is called the Clifford bundle:

$$
\mathrm{Cl}(E)=P_{\mathrm{SO}}(E) \times_{c l\left(\rho_{r, s}\right)} \mathrm{Cl}_{r, s}
$$

So $\mathrm{C}(E)$ as the quotient bundle:

$$
C \ell(E)=\left(\sum_{r=0}^{\infty} \otimes^{r} E\right) / I(E),
$$

where $I(E)$ is the bundle of ideals [2].

It is also clear that all fundamental concepts on Clifford algebras carries over to Clifford bundles. For example, $\mathrm{C} \ell(E)=\mathrm{Cl}^{0}(E) \oplus \mathrm{Cl}^{1}(E)$ corresponding to the even-odd decomposition of the algebras. 
These are the +1 and -1 eigen bundles of the bundle automorphism

$$
A: \operatorname{Cl}(E) \rightarrow \operatorname{Cl}(E) \quad[10] .
$$

These two definitions are the same since the fiber at $x \in E$ of $P_{\mathrm{SO}}(E) \times_{c l\left(\rho_{r, s}\right)} \mathrm{Cl}_{r, s}$ is $\mathrm{Cl}_{r, s}$ [10]. All notions familiar from Clifford algebras over real vector spaces carry over to Clifford bundles over manifolds. If $X$ is a pseudo-Riemannian manifold, we can construct the Clifford bundle $\mathrm{C}(\mathrm{TX})$ associated with the pseudoRiemannian form on the tangent bundle TX. We will also call this bundle $\mathrm{C} \ell(X)$, in case there is no confusion possible [10].

Definition 8. A smooth manifold endowed with a spin structure will be called a spin manifold [5].

Definition 9. (Spinor bundles). Let $X$ be a smooth manifold with a spin structure $\xi: P_{\mathrm{Spin}}(X) \rightarrow P_{\mathrm{SO}}(X)$. A real or complex spinor bundle of $X$ is a bundle of the form [1-3]

$$
S(X)=\left\{\begin{array}{l}
P_{\mathrm{Spin}}(X) \times_{\mu} L, \\
P_{\mathrm{Spin}}(X) \times_{\mu} L_{c},
\end{array}\right.
$$

where $L$ is a left module of $\mathrm{Cl}_{r, s}$ and $\mu$ : $\operatorname{Spin}(r, s) \rightarrow$ End $(L)$ is left-multiplication of elements in $\operatorname{Spin}(r, s)$, and where $L_{\mathbb{C}}$ is a complex left module for $\mathrm{C} \ell\left(\mathbb{R}^{n}\right) \otimes \mathbb{C}$ and $\mu$ : $\operatorname{Spin}(r, s) \rightarrow$ End $\left(L_{\mathbb{C}}\right)$ is the left-multiplication of elements in $\operatorname{Spin}(r, s)$. When $(r, s)=(1,3)$ defined as certain equivalence classes in appropriate sets, and a first definition for field of these object on living Minkowski space time was given [5].

Remark 3. The complexified left spin Clifford bundle denoted by

$$
\mathrm{Cl}_{\operatorname{Spin}_{1,3}}^{l}(X)=P_{\operatorname{Spin}_{1,3}}(X) \times_{l} \mathbb{C} \otimes \mathbb{R}_{1,3} \equiv P_{\operatorname{Spin}_{1,3}}(X) \times_{l} \mathbb{R}_{4,1}
$$

and the complexified right Clifford bundle by

$$
\mathrm{Cl}_{\operatorname{Spin}_{1,3}}^{r}(X)=P_{\operatorname{Spin}_{1,3}}(X) \times_{r} \mathbb{C} \otimes \mathbb{R}_{1,3} \equiv P_{\operatorname{Spin}_{1,3}}(X) \times_{r} \mathbb{R}_{4,1} \quad[5] .
$$

Remark 4. Taking, e.g., $L_{\mathbb{C}}=\mathbb{C}^{4}$ and $\mu_{\mathbb{C}}$ the $D^{(112,0)} \oplus D^{(0,1 \mid 2)}$ of $\operatorname{Spin}_{1,3} \cong \operatorname{SL}(2, \mathbb{C})$ in $\operatorname{End}\left(\mathbb{C}^{4}\right)$ and $L$ is covariant spinor bundle [5] (where $D$ is Dirac operator see the last sections).

If the module $L$ (or $L_{\mathbb{C}}$ ) is $\mathbb{Z}_{2}$ graded, the corresponding bundle is said to be $\mathbb{Z}_{2}$ graded.

Example 1. Consider $\mathrm{C} \ell\left(\mathbb{R}^{n}\right)$ as a module over itself by left multiplication $l$. The corresponding real spinor bundle $\mathrm{Cl}_{\text {Spin }}(X)=P_{\text {Spin }}(X) \times{ }_{l} \mathrm{Cl}\left(\mathbb{R}^{n}\right)$, then:

(i) $C \ell_{\text {Spin }}(X)$ is a "principal $C \ell\left(\mathbb{R}^{n}\right)$-bundle," i.e., it admits a free action of $\mathrm{C} \ell\left(\mathbb{R}^{n}\right)$ on the right.

(ii) There is a natural embedding $P_{\text {Spin }}(X) \subset C l_{\text {Spin }}(X)$, which comes from the embedding $\operatorname{Spin}_{n} \subset \mathrm{Cl}\left(\mathbb{R}^{n}\right)$. Hence, every real spinor bundle for $X$ can be captured from this one [2].

A similar remark holds for the complex case.

Of course, the bundle $\mathrm{Cl}_{\text {Spin }}(X)$ differs from the Clifford bundle $\mathrm{C} \ell(X)$. They can be compared as follows.

$$
\operatorname{Ad}: \operatorname{Spin}^{n} \rightarrow \operatorname{Aut}\left(C e\left(\mathbb{R}^{n}\right)\right)
$$

given by $\operatorname{Ad}_{g}(\phi)=g \varphi g^{-1}$ for $g \in \operatorname{Spin}^{n} \subset \mathrm{C} \ell\left(\mathbb{R}^{n}\right)$. Clearly $\operatorname{Ad}_{-1}=$ identity, and this representation come to acting $\mathrm{Ad}^{\prime}$ of $\mathrm{SO}_{n}$. One easily checks that $\mathrm{Ad}^{\prime}$ is just the representation $\mathrm{Ce}\left(\rho_{n}\right)$ given by

$$
\mathrm{Cl}(X)=P_{\text {Spin }}(X) \times_{\mathrm{Ad}} \mathrm{Cl}\left(\mathbb{R}^{n}\right) .
$$

Two spinor bundles of $X$ are equivalent if they are equivalent as bundles of $\mathrm{C} \ell(X)$-modules.

Real bundle, complex bundle, graded, and ungraded bundle of $\mathrm{Cl}(X)$ modules is called irreducible if at each $x$ fiber is irreducible as a (real or complex, graded or ungraded) module over $\mathrm{C} \ell\left(X_{x}\right)$ [2]. 
Then, when $X$ is a spin manifold;

(i) the components of $\mathrm{Cl}(X)=P_{\mathrm{Spin}}(X) \times_{\mathrm{Ad}} \mathrm{Cl}\left(\mathbb{R}^{n}\right)$ are homomorphism classes $[(\beta, \alpha)]$ of pairs $(\beta, \alpha)$, where $\beta \in P_{\text {Spin }}(X), \alpha \in \operatorname{Cl}\left(\mathbb{R}^{n}\right)$ and $(\beta, \alpha) \sim\left(\beta^{\prime}, \alpha^{\prime}\right) \Leftrightarrow \beta^{\prime}=\beta u^{-1}, \alpha^{\prime}=u \alpha u^{-1}$, for some $u \in$ Spin.

(ii) the components of $C \ell^{l}$ Spin $(X)$ are homomorphism classes of pairs $(\beta, \alpha)$ where $\beta \in P_{\text {Spin }}(X), \alpha \in \mathrm{C}\left(\mathbb{R}^{n}\right)$ and $(\beta, \alpha) \sim\left(\beta^{\prime}, \alpha^{\prime}\right) \Leftrightarrow \beta^{\prime}=\beta u^{-1}, \alpha^{\prime}=\alpha u^{-1}$, for some $u \in$ Spin.

(iii) the components of $C \ell^{r}$ Spin $(X)$ are homomorphism classes of pairs $(\beta, \alpha)$, where $\beta \in P_{\text {Spin }}(X), \alpha \in \mathrm{Cl}\left(\mathbb{R}^{n}\right)$ and $(\beta, \alpha) \sim\left(\beta^{\prime}, \alpha^{\prime}\right) \Leftrightarrow \beta^{\prime}=\beta u^{-1}, \alpha^{\prime}=\alpha u^{-1}$, for some $u \in$ Spin.

Proposition 14. There is a natural pairing $\operatorname{secCl}_{\mathrm{Spin}}^{l}(X) \times \operatorname{secCl}_{\mathrm{Spin}}^{r}(X)=\operatorname{secC} \ell(X)$.

Proof. Given $\delta \in \operatorname{secCl}_{\text {Spin }}^{l}(X), \rho \in \operatorname{secCl}_{\text {Spin }}^{r}(X)$ select representatives $(\beta, \alpha)$ for $\delta(x)$ and $(\beta, b)$ for $\rho(x)$, (with $\left.\beta \in \pi^{-1}(x)\right)$ and define $(\delta \rho)(x):=[(\beta, \alpha b)] \in \mathrm{Cl}(X)$. If alternative representatives $\left(\beta u^{-1}, u \alpha\right)$ and $\left(\beta u^{-1}, b u^{-1}\right)$ are chosen for $\delta(x)$ and $\rho(x)$, we have $\left(\beta u^{-1}, u \alpha b u^{-1}\right) \sim(\beta, \alpha b)$ and thus, $(\delta \rho)(x)$ is well define component of $\mathrm{Ce}(X)[5]$.

Let us now say a word about the $\mathbb{Z}_{2^{-}}$graded case. There is irreducible bundle of $\mathbb{Z}_{2^{-}}$graded modules over $\mathrm{Cl}(X)=\mathrm{Cl}^{0}(X) \oplus \mathrm{Cl}^{1}(X)$ and classes irreducible bundle of modules over $\mathrm{Cl}^{0}(X)$. Given a bundle $S(X)=$ $S^{0}(X) \oplus S^{1}(X)$ of the first kind, $S^{0}(X)$ is of the second. Given an $S^{0}(X)$ of the second kind, the bundle

$$
S(X)=\mathrm{C}(X)) \otimes_{\mathrm{C} e^{0}(X)} S O(X) \text { is of the first. }
$$

Suppose now that $n=2 \mathrm{~m}$ and $S_{\mathbb{C}}(X)$ is the irreducible complex spinor bundle of $X$. We will show clearly how to split $S_{\mathbb{C}}(X)$ into a direct sum:

$$
S_{\mathbb{C}}(X)=S_{\mathbb{C}}^{+}(X) \oplus S_{\mathbb{C}}^{-}(X)
$$

of $\mathrm{C}^{0}(X)$ modules. Interpreting $S_{\mathbb{C}}^{+}(X)$ as $S_{\mathbb{C}}^{0}(X)$ and $S_{\mathbb{C}}^{-}(X)$ as $S_{\mathbb{C}}^{1}(X)$, or the other way around, gives a $\mathbb{Z}_{2^{-}}$ graded module structure to $S_{\mathbb{C}}(X)$.

There is a similar construction in the real case.

$$
S(X)=S^{+}(X) \oplus S^{-}(X) \quad[2] .
$$

Recall that every module for $\mathrm{C} \ell\left(\mathbb{R}^{n}\right)$ is a direct sum of irreducible ones, and there are at most two homomorphism classes of irreducible modules [2].

Proposition 15. If $S(X)$ is a real spinor bundle of $X$, then $S(X)$ is a bundle of modules over the bundle of algebras $\mathrm{Cl}(X)$. In particular, the sections of the spinor bundle are a module over the sections of the Clifford bundle [5].

Proof. See [2].

Remark 5. The corresponding fact holds in the complex and $\mathbb{Z}_{2}$-graded cases and Sections of $S(X)$ are called spinors.

Definition 10. The dual spinor bundle $S^{*}(X)$ is a real or a complex spinor bundle

$$
S^{*}(X)=\left\{\begin{array}{l}
P_{\text {Spin }}(X) \times_{\mu} L^{*}, \\
P_{\text {Spin }}(X) \times_{\mu} L_{c}^{*},
\end{array}\right.
$$

where $L^{*}$ is a right module of $\mathrm{Cl}_{r, s}$ and $\mu: \operatorname{Spin}(r, s) \rightarrow \operatorname{End}(L)$ is the representation given by right-multiplication of (inverse) elements in $\operatorname{Spin}(r, s)$, and where $L_{\mathbb{C}}^{*}$ is a complex right module for $\mathrm{Cl}\left(\mathbb{R}^{n}\right) \otimes \mathbb{C}$ and $\mu$ : $\operatorname{Spin}(r, s) \rightarrow \operatorname{End}\left(L_{\mathbb{C}}\right)$ is the representation given by right-multiplication of inverse elements in $\operatorname{Spin}(r, s)$ [5].

Definition 11. If $U$ is a normal bundle of the Grassmannian. Then, if $s^{*} U=S_{(X)}$ the spinor bundle on $Q_{2 k+1}$. Its rank is $2^{k}$. We call $s^{\prime *} U=S^{\prime}{ }_{(X)}$ and $s^{\prime \prime *} U \simeq S^{\prime \prime}{ }_{(X)}$ the two spinor bundles on $Q_{2 k}$. Their rank is $2^{k-1}$. 
If $f$ is an automorphism of $Q_{2 k}$ that exchanges the two families of $k$-planes, we have

$$
f^{*} S_{(X)}^{\prime} \simeq S^{\prime \prime}(X) \text { and } f^{*} S^{\prime \prime}(X) \simeq S_{(X)}^{\prime} .
$$

Clear that $S_{(X)}$ (spinor bundles) on all quadrics $Q$ are homogeneous, i.e., $f^{*} S_{(X)} \simeq S_{(X)}$ for all $f \in \operatorname{Aut}(Q)_{0}$, where $\operatorname{Aut}(Q)_{0}$ is the connected component of the identity in $\operatorname{Aut}(Q)$.

\section{Theorem 16.}

(i) Let $S_{(X)}^{\prime}, S^{\prime \prime}{ }_{(X)}$ are spinor bundles on $Q_{2 k}$, and let $i: Q_{2 k-1} \rightarrow Q_{2 k}$ be a smooth hyper plane section. Then $i^{*} S_{(X)}^{\prime} \simeq i^{*} S^{\prime \prime}{ }_{(X)} \simeq S_{(X)}$, where $S_{(X)}$ is the spinor bundle on $Q_{2 k-1}$.

(ii) Let $S_{(X)}$ is spinor bundle on $Q_{2 k+i}$, and let $i: Q_{2 k} \rightarrow Q_{2 k+i}$ be a smooth hyperplane section.

Then, $i^{*} S_{(X)} \simeq S_{(X)}^{\prime} \oplus S^{\prime \prime}(X)$, where $S_{(X)}^{\prime}$ and $S^{\prime \prime}{ }_{(X)}$ are the spinor bundles on $Q_{2 k}$ [11].

Example 2. Two embeddings $s^{\prime}: Q_{4} \rightarrow \operatorname{Gr}(l, 3)$ and $S^{\prime \prime}: Q_{4} \rightarrow \operatorname{Gr}(l, 3)$ are isomorphisms from the definition of spinor bundles. So $S_{(X)}$ on $Q_{4}$ are the normal bundle and the dual of the quotient bundle.

The embedding $S: Q_{3} \rightarrow \operatorname{Gr}(l, 3)$ corresponds to a hyperplane section. If $S_{(X)}$ is the spinor bundle on $Q_{3}$, then $S_{(X)}^{2} S_{(X)}^{*}=T Q_{3}$. In fact, $T Q_{4} / Q_{3} \simeq S_{(X)}^{*} \oplus S_{(X)}^{*} \simeq S_{(X)}^{2} S_{(X)}^{*} \oplus$ (1) and the exact sequence splits

$$
0 \rightarrow T Q_{3} \rightarrow T Q_{4} / Q_{3} \rightarrow(1) \rightarrow 0 .
$$

On $Q_{2}$ the two of $S_{(X)}$ are the duals of the two line bundles corresponding to two skew-lines on $Q_{2}$. On $Q_{1} \simeq \mathbb{P}^{1}$, then the spinor bundle can defined to be $O_{\mathbb{P}^{1}(-1)}$ [11].

Corollary 3. Let $l \subset Q_{n}(n>3)$ be a line and let $S_{(X)}$ on $Q_{n}$ [11]. Then

$$
\left.S_{X}\right|_{l}=O_{l}^{\oplus 2\left[\frac{(n-3)}{2}\right]} \oplus O_{l}(-1)^{\oplus 2\left[\frac{(n-3)}{2}\right] .}
$$

\section{Relations between spinor bundles and spinor structures}

For every $x \in M$, the spinor representation $\tau_{x}: C \ell\left(g_{x}\right) \rightarrow$ End $\Sigma_{x}$ defines the real line $a\left(\tau_{x}\right)$ and the circle $c\left(\tau_{x}\right)[12]$. The set

$$
\alpha(\tau)=\bigcup_{x \in M} \alpha\left(\tau_{x}\right) \subset \operatorname{Hom}\left(\Sigma, \overline{\Sigma^{*}}\right)=\Sigma^{*} \otimes \overline{\Sigma^{*}}
$$

has the structure of a real line bundle over $M$. The set

$$
c(\tau)=\bigcup_{x \in M} c\left(\tau_{x}\right) \subset \operatorname{Hom}(\Sigma, \bar{\Sigma})=\stackrel{\Sigma}{\Sigma}^{*} \otimes \bar{\Sigma}
$$

has the structure of a bundle of circles over $M$ : It is a principal $U(1)$-bundle. If this bundle is trivial, i.e., if it has a (global) section $C: M \rightarrow c(\tau)$, then the real line bundle over $M$ defined as follows:

$$
b(\tau, C)=\bigcup_{x \in M} b\left(\tau_{x}, C(x)\right) \subset \operatorname{Hom}\left(\Sigma, \Sigma^{*}\right)=\Sigma^{*} \otimes \Sigma^{*} .
$$

Proposition 17. Let $(M, \mathrm{~g})$ be an smooth Riemannian manifold with $(V, h)$ as the local model.

(i) There corresponds a Clifford ${ }^{c}$ structure to every spinor bundle such that the associated spinor bundle is isomorphic to $\tau: \mathrm{Cl}(\mathrm{g}) \rightarrow$ End $\Sigma[3]$.

(ii) The Clifford ${ }^{c}$ structure can be reduced to a spin structure iff the line bundle above is trivial.

(iii) The Clifford ${ }^{c}$ structure can be reduced to a Clifford structure iff the bundle of circles above is trivial.

(iv) The resulting Clifford structure can be reduced to a spin structure iff the real line bundle above is trivial $[13,14]$. 
Proof. We already know that $\operatorname{Hom}_{C \ell M_{\mathrm{C}}}(S, \bar{S})$ is a complex line bundle: each fiber contains a $\mathrm{Ce}\left(T_{p} M_{\mathrm{C}}\right)$-linear isomorphism $\tau_{p}: S_{p} \rightarrow \bar{S}_{p}$ and by irreducibility of $S_{p}$ and $\bar{S}_{p}$ and Schur's Lemma any Ce( $\left(T_{p} M_{\mathbb{C}}\right)$-linear map $\tau_{p}^{\prime}: \bar{S}_{p} \rightarrow S_{p}$ satisfies $\tau_{p}^{\prime} \circ \tau_{p}=\lambda / S_{p}$ for some $\lambda \in \mathbb{C}$. In case of a spin structure $\tau$ defines a non-vanishing section in $\operatorname{Hom}_{C \ell M_{\mathrm{C}}}(S, \bar{S})$, hence

$\operatorname{Hom}_{C e M_{\mathrm{C}}}(S, \bar{S})$ is trivial. Conversely, if this bundle is trivial and if $\tilde{\tau}^{\prime}$ is a nonvanishing section, then $\tilde{\tau}^{\prime 2}=\lambda / s$ for some nonvanishing map $\lambda \in \mathbb{C}^{\infty}(M, \mathbb{C})$. But $\lambda(p) \tilde{\tau}_{p}(v)=\tilde{\tau}_{p} \circ \tilde{\tau}_{p} \circ \tilde{\tau}_{p}(v)=\tilde{\tau}(\lambda(p) v)=\overline{\lambda(p)} \tilde{\tau}(v)$, i.e., $\lambda \in \mathbb{C}^{\infty}(M)$ is real valued, and replacing $\tilde{\tau}$ by $\tau=|\lambda|^{-1 / 2} \tilde{\tau}$, we obtain a structural map.

Now given an irreducible complex spinor bundle $S$ and a structural map $\tau$, we can choose a Riemannian structure compatible with Clifford multiplication and such that $\tau$ is an isometry.

Definition 12. Suppose $E$ is a smooth Riemannian vector bundle over a manifold $X$ and that $\xi: P_{\text {Spin }}(E) \rightarrow$ $P_{\mathrm{SO}}(E)$ is a spin structure on $E$. Then, of course, any connection on $P_{\mathrm{SO}}(E)$ can be lifted via $\xi$ to a connection on $P_{\text {Spin }}(E)$, and this, in turn, defines a connection on the associated spinor bundles [2].

We give two equivalent definitions of a connection on a principal $G$-bundle with projection $P \stackrel{\pi}{\rightarrow} X$.

First, we define the vertical tangent space $T_{p}^{v} P$ at $p$ as the subspace of $T_{p} P$, which is tangent to the fiber of the projection $P \stackrel{\pi}{\rightarrow} X$. A connection on $P \stackrel{\pi}{\rightarrow} X$ is a smoothly varying family of linear subspaces $\left(T_{p}^{H}\right)_{p \in P}$ of the tangent bundle $T P$, which is everywhere complementary to the vertical distribution $\left(T_{p}^{v} V\right)_{p \in P}$ and which is invariant under the action of the group $G$. The distribution $\left(T_{p}^{H}\right)_{p \in P}$ is called the horizontal distribution. Note that

$$
T P=T^{V} P \oplus T^{H} P \quad[12] .
$$

Second, to give a connection on $P \stackrel{\pi}{\rightarrow} X$ is to give a connection 1-form $\omega$ on $P$ with values in $g$ satisfying two conditions.

It transforms by the adjoint action, i.e., for any $g$ in $G, p$ in $P$ and any $y$ in $T_{p} P$, we have

$$
\omega_{p} g(y g)=g^{-1} \omega_{p}(y) g .
$$

For any $a$ in $g$, the associated vector field $V a$ on $P$ defined by the tangent vector of the curve $p e^{t a}$ at any $p \in P$. It holds that $\omega\left(V_{a}\right)=a$.

Note that the horizontal distribution can be recaptured by taking the kernel of the connection 1-form assigned to it.

Lemma 18. Given a Euclidean connection on a real vector bundle E, there is a canonical orthogonal connection (i.e., the decomposition $T P=T^{V} P \oplus T^{H} P$ is Euclidean) on its orthonormal frame bundle $P \stackrel{\mathrm{SO}(n)}{\rightarrow} X$. Reversely, any orthogonal connection on the frame bundle $P \stackrel{S O(n)}{\rightarrow} X$ induces a Euclidean connection on E. Furthermore, these operations are inverse to each other [4].

Lemma 19. Given a Lie group $G$ and a connection on a principal $G$-bundle $P \stackrel{\pi}{\rightarrow} X$, there is an induced connection on any vector bundle $P \times_{G} V$ coming from a linear representation $G \rightarrow G L(V)$ [4].

The curvature g-valued 2-form $\Omega$ is

$$
\Omega=\mathrm{d} \omega+[\omega, \omega]
$$

Example 3. (Orthogonal connections). Let $P=P_{\mathrm{SO}}(E)$, where $E$ is a smooth, oriented Riemannian vector bundle. The Lie algebra $\mathrm{SO}_{n}$ of real, skew-symmetric $n \times n$-matrices. Hence, a connection 1-form $\omega$ can be considered as an $n \times n$-matrix of 1-forms $\omega=\left(\omega_{i j}\right)$, where $\omega_{i j}=-\omega_{j i}^{\prime \prime}$. 
The corresponding curvature is a matrix of 2-forms $\Omega=\Omega_{i j}$, where

$$
\Omega_{i j}=\mathrm{d} \omega_{i j}+\sum_{k=1}^{n} \omega_{i k} \Lambda \omega_{k j}
$$

Suppose that $\mu=\left(e_{1}, \ldots, e_{n}\right)$ is just a section of $P_{\mathrm{SO}}(E)$ over $U \subseteq X$, and it can be lifted to a section $\tilde{\mu}$ of $P_{\text {Spin }}$ (E) over $U$. There are two possible such liftings. They satisfy the relation:

$$
\xi \circ \tilde{\mu}=\mu
$$

The connection 1-form on $P_{\text {Spin }}(E)$ is just the lift $\xi^{*} \omega$ (the pull down) of the connection 1-form $\omega$ on $P_{\mathrm{SO}}(E)[2]$.

Theorem 20. Let $\omega$ be the connection i-form on $P_{\mathrm{SO}}(E)$ and let $S(E)$ be any spinor bundle associated to $E$. Then, the covariant derivative $\nabla^{S}$ on $S(E)$ is given locally by the formula [2]:

$$
\nabla^{s}=\frac{1}{2} \sum_{i<j} \tilde{\omega}_{i j} \otimes e_{i} e_{j} .
$$

where $\mu=\left(e_{1}, \ldots, e_{n}\right)$ is a local section of $P_{\mathrm{SO}}(E), \omega=\mu^{*}(\omega)$, and where $E=\left(\sigma_{1}, \ldots, \sigma_{n}\right)$ is a local section of $P_{\mathrm{SO}}(S(E))$ determined by $\omega$.

Theorem 21. Let $\Omega$ be the curvature 2-form on $P_{\mathrm{SO}}(E)$ and let $S(E)$ be any spinor bundle associated to $E$. Then, the curvature $\mathcal{R}$ by the formula $S$ of $S(E)$ is locally given by

$$
R^{s}=\frac{1}{2} \sum_{i<j} \widetilde{\Omega}_{i j} \otimes e_{i} e_{j},
$$

where $\mu=\left(e_{1}, \ldots, e_{n}\right)$ is a section of $P_{\mathrm{SO}}(E), \widetilde{\Omega}=\mu^{*}(\Omega)$ and $\sigma$ is any section of $S(E)$ [2].

Then, any two tangent vectors $\boldsymbol{V}$ and $\boldsymbol{W}$ at $x \in X$, the curvature transformation $\mathcal{R}_{V, W}^{S}: S\left(E_{x}\right) \rightarrow S\left(E_{x}\right)$ is

$$
R_{V, W}^{S}=\frac{1}{2} \sum_{i<j}\left\langle R_{V, W}\left(e_{i}, e_{j}\right)\right\rangle e_{i} e_{j},
$$

where $\mathcal{R}_{v, w}$ is the curvature transformation of $E_{x}$.

Dirac operator $D$ acting on sections of spinor bundle $\left(S_{(X)}\right) \Sigma \rightarrow M$ is globally defined as follows.

Definition 13. (Dirac operator). Let $U_{l}$ be an open subset of $M$ and let $e=\left(e_{\mu}\right)_{\mu=1, \ldots, m}$ be a field of (not necessarily orthonormal) frames on $U_{l}$. For every $p \in U_{l}$, the components of the metric tensor $g$ with respect to $e$ at $p$ are $g_{\mu v}(p)=g\left(e_{\mu}(p), e_{\nu}(p)\right)$ and there is the inverse $g^{\mu v}(p)$ of $g_{\mu v}(p)$. The restriction of the Dirac operator to $U_{l}$ is [2] expressed as follows:

$$
D=g^{\mu v} \tau\left(e_{\mu}\right) \nabla_{e_{v}} .
$$

The Dirac operator on $M$ well defined by its restrictions to the sets ( $U$ ) providing an open cover of $M$. $\nabla^{T M}$ is connection on the spinor bundle to be metric, but may have torsion.

Definition 14. A Dirac bundle is a bundle $S$ over a Riemannian manifold $X$ of left modules over $\mathrm{Cl}(X)$ together with a Riemannian metric and connection on $S$ with properties:

$$
e_{1} \sigma_{1}, e_{2} \sigma_{2}=\sigma_{1}, \sigma_{2} \quad \text { and } \quad \nabla(\varphi \sigma)=(\nabla \varphi) \sigma+\varphi(\nabla \sigma) \text { for all } \varphi \in \Gamma(\mathrm{Cl}(X)), \quad \sigma \in \Gamma(S) \text {. }
$$

The operator $D$ is elliptic if the linear map $\sigma_{\xi}(D): E_{x} \rightarrow E_{x}$ is an isomorphism for all $\xi \neq 0$. 
Lemma 22. If $D$ is the Dirac operator of the bundle $S$ defined above. Then, for any $\xi \in T^{*}(X) \cong T(X)$, we have that

$$
\begin{gathered}
\sigma_{\xi}(D)=i \xi, \\
\sigma_{\xi}\left(D^{2}\right)=\|\xi\|^{2},
\end{gathered}
$$

where the symbol on the right denotes Clifford multiplication by the vector $\xi$ and the scalar $\left\|\xi^{2}\right\|$. In particular, both $D$ and $D^{2}$ are elliptic operators.

Proof. See [2].

Theorem 23. Let $X$ be a complete Riemannian manifold and if $D$ is the Dirac operator of Dirac bundle $S$ over $X$. Then, the closure of $D$ in $L^{2}(S)$ is a self-adjoint operator. Furthermore, $\operatorname{ker}(D)=\operatorname{ker}\left(D^{2}\right)$ on $L^{2}(S)$, where $L$ is a canonical bundle map $L: \mathrm{Cl}(X) \rightarrow \mathrm{Cl}(X)$ defined by $L(\varphi)=-\sum e_{j} \varphi e_{j}[2]$.

Lemma 24. Let $D$, $S$ and $X$ be as above. Then for any $f \in \mathrm{C}^{\infty}(X)$ and any $\varphi \in \Gamma(S) \subset \mathrm{C}^{\infty}(X)$, we have that

$$
D(f \varphi)=(\operatorname{grad} f) \varphi+f D \varphi .
$$

Proof. $\left.D(f \varphi)=\sum e_{j} \cdot \nabla e_{j}(f \varphi)=\sum e_{j}\left\{\left(e_{j} f\right) \varphi+f \nabla e_{j} \varphi\right\}=\sum\left(\left(e_{j} f\right) e_{j} \cdot \varphi\right)+f D \varphi\right)=(\operatorname{grad} f) \cdot \varphi+f D \varphi[2]$.

Having discussed Dirac bundles in general terms, it is now time to look hard at some important examples. We begin with the basic ones.

Example 4. (An historical case). Let $X=R^{n}$, Euclidean $n$-space, and let $S=R^{n} \times V$, where $V$ some module for $\mathrm{Cl}_{n}$. Here, $D$ is a constant on $V$-valued functions [2]

$$
D=\sum_{k=1}^{n} y_{k} \frac{\partial}{\partial x_{k}}
$$

and each $\gamma_{k}$ is a linear map $\gamma_{k}: V \rightarrow V$ and $\gamma_{j} \gamma_{k}+\gamma_{k} \gamma_{j}=-2 \delta_{j k}$.

This particular operator has historical roots in physics. In the 1920s [2], the physicist P.A.M. Dirac was searching for a Lorentz-invariant first-order differential operator whose square would be the Klein-Gordon operator. Thus, he was essentially led to search for a first-order operator $D$ of the form above, which satisfied the equation $D^{2}=\Delta$, where $\Delta=-\sum \partial^{2} / \partial x^{2}$ is the positive Laplacian in $\mathbb{R}^{n}$. Realizing that the $\gamma_{k} \mathrm{~s}$ must be matrices, he was led immediately by this equation to the aforementioned relations, which we recognize now as the generating relations of a representation of $\mathrm{Cl}_{n}$.

Let $n=1$, so that $C \ell_{1}=V=\mathbb{C} \cong \mathbb{R}^{2}$. Then we have $[3,8]$

$$
D=i \frac{\partial}{\partial x_{1}}
$$

the generator of a basic semi-group of unitary operators on $L^{2}$.

Let $n=2$, so that $\mathrm{Cl}_{2}=V=\mathbb{H} \cong \mathbb{C} \otimes \mathbb{C}$. The construction of $\mathbb{H}$ into $\mathbb{C} \otimes \mathbb{C}$ is natural and corresponds to the $\mathbb{Z}_{2}$-grading $C \ell_{2}^{0} \otimes C \ell_{2}^{1}[3]$

$$
D=e_{1} \frac{\partial}{\partial x_{1}}+e_{2} \frac{\partial}{\partial x_{2}}
$$

has the form $D=\left(\begin{array}{cc}0 & -\frac{\partial}{\partial z} \\ \frac{\partial}{\partial \bar{z}} & 0\end{array}\right)$, where $\partial / \partial \bar{z}=\partial / \partial x_{1}+i \partial / \partial x_{2}$. 
Let $n=3$, so that $C \ell_{3}=\mathrm{H} \otimes \mathbb{H}$ and $V=\mathbb{H}$. Cl $\ell_{3}$ has two representations on $\mathbb{H}$ given as Identify $\mathbb{R}^{3}$ with $\operatorname{Im}(\mathbb{H})$, by letting $i, j$, and $k$ act on either the right or the left in $\mathbb{H}$. On the left, we get Dirac operator on $\mathbb{H}$ :

$$
D=i \frac{\partial}{\partial x_{1}}+j \frac{\partial}{\partial x_{2}}+k \frac{\partial}{\partial x_{3}} .
$$

Let $n=4$, so that $\mathrm{Cl}_{4}=\mathbb{H}(2)$ and $V=\mathbb{H}^{2}=\mathbb{H} \otimes \mathbb{H}$. To describe the full Dirac operator, we consider first the following $\mathbb{H}$ under the basis $(1, i, j, k)$ :

$$
\frac{\partial}{\partial \bar{q}}=\frac{\partial}{\partial x_{0}}+i \frac{\partial}{\partial x_{1}}+j \frac{\partial}{\partial x_{2}}+k \frac{\partial}{\partial x_{3}}, \quad \frac{\partial}{\partial q}=\frac{\partial}{\partial x_{0}}+i \frac{\partial}{\partial x_{1}}+j \frac{\partial}{\partial x_{2}}+k \frac{\partial}{\partial x_{3}},
$$

then $D=\left(\begin{array}{cc}0 & -\frac{\partial}{\partial q} \\ \frac{\partial}{\partial \bar{q}} & 0\end{array}\right)$.

Thus, left multiplication be represented by complex $2 \times 2$-matrices $\sigma_{0}, \sigma_{2}$ and $\sigma_{3}$ respectively, then the operator $\partial / \partial \bar{q}$ becomes

$$
\frac{\partial}{\partial \bar{q}}=\frac{\partial}{\partial x_{0}}+\sigma_{1} \frac{\partial}{\partial x_{1}}+\sigma_{2} \frac{\partial}{\partial x_{2}}+\sigma_{3} \frac{\partial}{\partial x_{3}} .
$$

The matrices $\sigma_{\mathrm{k}}$ can be chosen to be the classical Pauli matrices:

$$
\sigma_{1}=\left(\begin{array}{cc}
i & 0 \\
0 & -i
\end{array}\right), \quad \sigma_{2}=\left(\begin{array}{cc}
0 & 1 \\
-1 & 0
\end{array}\right), \quad \sigma_{3}=\left(\begin{array}{ll}
0 & i \\
i & 0
\end{array}\right)
$$

Note that these matrices generate the fundamental representation of $\mathrm{Cl}_{3}$ in complex form $[2,3]$.

Example 5. (The Clifford bundle). Let $S=\mathrm{C \ell}(X)$ with its canonical Riemannian connection, and view $\mathrm{C} \ell(X)$ as a bundle of left modules over itself by left Clifford multiplication. The Dirac operator D in this case is a square root of the classical Hodge Laplacian [2].

Example 6. (The spinor bundles). Suppose $X$ is a spin manifold with a spin structure on its tangent bundle. Let $S_{(X)}$ be any spinor bundle associated with $T(X)$. Then, $S_{(X)}$ is a bundle of modules over Ce(X), and $S_{(X)}$ carries a canonical Riemannian connection, which has property of Definition 14 . The Dirac operator in this case was first written down by Atiyah and Singer in their work on the Index theorem. Finding this operator was a major accomplishment, and for this reason, we shall call it the Atiyah-Singer operator $[2,15]$.

Notation. For spin manifolds $X$ of even dimension, we shall denote the (unique) irreducible complex spinor bundle by $\$ \mathbb{C}$; and when $\operatorname{dim}(X) \neq 3(\bmod 4)$, we denote the irreducible real spinor bundle by $\$$.

In both cases, the Atiyah-Singer operator will be written as $\not$.

These basic examples each generate large families of new examples by the following construction.

Let $S$ and $E$ be a given Dirac bundle with connection $\nabla^{\mathrm{s}}$ and $\nabla^{E}$ over a Riemannian manifold $X$. Then, the tensor product $S \otimes E$ is again a bundle of left modules over $\mathrm{Cl}(X)$, where for $\varphi \in \operatorname{Cl}(X), \sigma \in S, e \in E$, $\varphi .(\sigma \otimes e)=(\varphi, \sigma) \otimes e[2]$.

Furthermore, we can equip $S \otimes E$ with the canonical tensor product connection, $\nabla=\nabla^{\mathrm{s}} \otimes \nabla^{E}$, which is defined on sections of the form $\sigma \otimes e$ by the formula:

$$
\nabla(\sigma \otimes e)=\left(\nabla^{\mathrm{s}} \sigma\right) \otimes e+\sigma \otimes\left(\nabla^{E} e\right) .
$$




\section{Conclusion}

The researches have explained how Riemannian geometry, with the theory of spinor fiber bundle, fits into the general meaning of principal fiber bundles. We see that the spinor bundles defined as vector bundles whose fibers carry spinor representations of the Clifford algebras $\mathrm{Cl}(\mathrm{g})$, spinor fields are sections of spinor bundles.

With this constructive classification tool, we have investigated the usefulness of spinor structures of Clifford algebra. We have also given an explicit description of an important physical applications of spinor bundle and used a spinor connection and the constant Dirac matrices of special relativity to define the Dirac operator using the Clifford algebra of space-time and Dirac operation.

Acknowledgments: The author would like to thank the Deanship of Scientific Research, Qassim University for funding publication of this project.

Conflict of interest: The author states no conflict of interest.

\section{References}

[1] J. J. Venselaar, Spinors and Dirac Operators, Caltech, California, USA, 2007.

[2] H. B. Lawson and M.-L. Michelsohn, Spin Geometry, Princeton University, New Jersey, USA, 1989.

[3] H. Schröder, On the definition of geometric Dirac operators, https://arxiv.org/abs/math/0005239, (2000).

[4] J. M. Bismut, Introduction to the Index Theory, University of Paris, France, 1986.

[5] A. R. Mosona and A. W. Rodrigues, The Fundle of Algebraic and Dirac-Hestenes Spinor Field, 13083-970 Campinas, SP Brazil, 2004.

[6] S. Morrison, Classifying spinor structures, Master's thesis, University of New South Wales, https://arxiv.org/abs/math$\mathrm{ph} / 0106007,(2001)$.

[7] N. Steenrod, The Topology of Fibre Bundles, Princeton University, USA, 1999.

[8] K. Wernli, Lecture notes on spin geometry, arXiv:1911.09766v1, (2019).

[9] L. Claessens, Field theory from a bundle point of view, Belgium, 2011, https://www.yumpu.com/en/document/view/ 33648732/field-theory-from-a-bundle-point-of-view.

[10] J. L. Koszul, Lectures on Fibre Bundles and Differential Geometry, Bombay, 1960.

[11] G. Ottaviani, Spinor bundles on quadrics, Trans. Amer. Math. Soc. 307 (1988), no. 1, 301-316.

[12] A. Trautman, Connections and the Dirac operator on spinor bundles, J. Geom. Phys. 58 (2007), 238-252.

[13] C. Kassel, Homology and Cohomology of Associative Algebras, Notes in the Advanced School on Non-commutative Geometry at ICTP, Trieste, Italy, 2004.

[14] T. Friederish and A. Trautman, Clifford Structures and Spinor Bundles, Berlin, Germany and Warsaw, Poland, 1997.

[15] M. F. Atiyah, R. Bott, and A. Shapiro, Clifford modules, Topology 3 (1964), 3-38. 